\title{
Cell division autoantigen 1 plays a profibrotic role by modulating downstream signalling of TGF $-\beta$ in a murine diabetic model of atherosclerosis
}

\author{
Y. Pham • Y. Tu • T. Wu • T. J. Allen • A. C. Calkin • \\ A. M. Watson • J. Li • K. A. Jandeleit-Dahm • \\ B-H. Toh • Z. Cao • M. E. Cooper • Z. Chai
}

Received: 30 July 2009 /Accepted: 27 August 2009/Published online: 22 October 2009

(C) Springer-Verlag 2009

\begin{abstract}
Aims/hypothesis Excess accumulation of vascular extracellular matrix $(\mathrm{ECM})$ is an important pathological process in cardiovascular diseases including diabetes-associated atherosclerosis. We explored how a recently identified molecule, cell division autoantigen 1 (CDA1), influences the profibrotic TGF- $\beta$ pathway leading to vascular ECM accumulation.

Methods Expression levels of genes encoding for CDA1, TGF- $\beta$ and connective tissue growth factor (CTGF) were examined in aorta from Apoe $e^{-/}$mice with or without diabetes. We used retroviral and adenoviral constructs to knockdown or overexpress $T$ spyl2, the gene encoding CDA1, in mouse vascular smooth muscle cells (VSMCs) with or without TGF- $\beta$ treatment in order to demonstrate the role of CDA1 in TGF- $\beta$ signalling.

Results In vivo studies indicated that the mRNA levels of CDA1-encoding gene $T_{s p y l 2}$ and protein levels of CDA1 were elevated in the aorta of diabetic $A p o e^{-/-}$mice, accompanied by increased levels of $\operatorname{Tg} f-\beta$ (also known as
\end{abstract}

Electronic supplementary material The online version of this article (doi:10.1007/s00125-009-1555-9) contains supplementary material, which is available to authorised users.

Y. Pham $\cdot$ Y. Tu $\cdot$ T. Wu $\cdot$ T. J. Allen • A. C. Calkin

A. M. Watson $\cdot \mathrm{J}$. Li $\cdot$ K. A. Jandeleit-Dahm $\cdot$ Z. Cao $\cdot$

M. E. Cooper $\cdot$ Z. Chai $(\triangle)$

Diabetes and Metabolism Division,

Baker IDI Heart and Diabetes Institute,

75 Commercial Rd,

Melbourne, Victoria 3004, Australia

e-mail: zhonglin.chai@bakeridi.edu.au

B.-H. Toh

Autoimmunity Laboratory, Centre for Inflammatory Diseases,

Department of Medicine, Monash University,

Clayton, Victoria, Australia
$T g f b 1), C \operatorname{tg} f$ and ECM accumulation. In vitro studies in vascular cells showed that TGF- $\beta$ treatment rapidly increased CDA1 protein levels, which then amplified TGF- $\beta$ signalling leading to upregulation of ECM genes. Knockdown of CDA1-encoding gene Tspyl2 to reduce cellular CDA1 level markedly attenuated TGF- $\beta$-stimulated MAD homologue 3 (drosophila; SMAD3) phosphorylation and transcriptional activities. CDA1 overproduction increased and Tspyl2 knockdown decreased expression of TGF- $\beta$ receptor type I, $T \beta r I$ (also known as $T g f b r 1$ ), but not TGF$\beta$ receptor type II, $T \beta r I I$ (also known as $T g f b r 2$ ), providing a mechanism for CDA1's action in modulating TGF- $\beta$ signalling. Knockdown of CDA1-encoding gene Tspyl2 also blocked the profibrotic effect of TGF- $\beta$ in VSMCs.

Conclusions/interpretation CDA1 plays an important role in vascular ECM accumulation by amplifying TGF- $\beta$ signalling. This is critical for the profibrotic effect of TGF- $\beta$ in the vasculature. $\mathrm{CDA} 1$ is therefore a potential target for attenuating vascular ECM accumulation caused by enhanced TGF- $\beta$ action, as seen in diabetic atherosclerosis.

Keywords Atherosclerosis - CDA1 - Diabetes · Fibrosis . TGF- $\beta \cdot$ Vascular ECM accumulation
Abbreviations
CDA1 Cell division autoantigen 1
CTGF Connective tissue growth factor
ECM Extracellular matrix
ERK Extracellular signal-regulated protein kinase
MAPK Mitogen-activated protein kinase
siRNA Short interfering RNA
SMAD MAD homologue 3 (drosophila)
VSMC Vascular smooth muscle cell 


\section{Introduction}

Cardiovascular disease usually arises from vascular dysfunction as a result of atherosclerosis and/or thrombosis, and is clinically associated with diabetes [1]. Excess accumulation of extracellular matrix (ECM) in the vasculature appears to play an important role in the development and progression of the vasculopathy seen in diabetes [2-6]. However, the underlying molecular mechanisms have not been fully delineated.

TGF- $\beta$ is the major growth factor implicated in vascular matrix accumulation via the SMAD signalling pathway $[6,7]$. TGF- $\beta$-dependent and -independent signalling pathways involving R-SMAD activation are the major pathways via which various pathological stimuli that appear to be enhanced in the diabetic milieu, e.g. TGF- $\beta$, angiotensin II, AGE, glucose and mechanical stretch, stimulate blood vessel ECM accumulation and remodelling [6]. It has been postulated that targeting downstream signalling of TGF- $\beta$ could be beneficial by reducing ECM accumulation [8]. Indeed, blockade of the renin-angiotensin system with drugs such as ACE inhibitors has been shown to reduce fibrosis, partly via TGF- $\beta$-dependent pathways, and has been reported to attenuate atherosclerosis in the setting of diabetes [4]. However, targeting ECM accumulation in the atherosclerotic plaque at a later stage remains controversial since in certain contexts, such as in the fibrous cap, TGF- $\beta$ may act paradoxically to reduce the risk of subsequent acute plaque rupture [9-11].

Cell division autoantigen 1 (CDA1) is a molecule that we identified and named to reflect our initial finding that it arrested cell growth when overexpressed in HeLa cells [12]. Its anti-proliferative action is supported by our further findings as well as by those of other groups [13-15]. We have shown that CDA1 is able to activate the extracellular signal-regulated protein kinase (ERK)/mitogen-activated protein kinase (MAPK) pathway and transcriptionally upregulate the cyclin-dependent kinase inhibitor, p21 Wafl/Cip1 [13]. Both the latter and ERK/MAPK are known to be regulated by TGF- $\beta$ [16-21]. CDA1 has been shown to be upregulated by TGF- $\beta$ and to increase luciferase activities of TGF- $\beta$ reporter constructs in some lung cancer cell lines [22-25]. These findings suggest that CDA1 may be involved in TGF- $\beta$ signalling and play a role in mediating the biological functions of TGF- $\beta$. TGF- $\beta$ upregulation is thought to be a pathological event in diabetes, leading to prominent ECM accumulation and thereby promoting various diabetic complications, including nephropathy and macrovascular diseases [26, 27].

Here, we not only show overexpression of the CDA1encoding gene Tspyl2 in diabetic Apoe ${ }^{-/-}$mice, which exhibit accelerated atherosclerosis, but also identify a novel role for CDA1 in modulating TGF- $\beta$ signalling in vascular smooth muscle cells (VSMCs), which leads to ECM gene expression. Thus, these data show that CDA1 is an appropriate and potentially attractive molecular target to block the profibrotic effect of TGF- $\beta$, thus preventing, retarding or treating diabetes-accelerated vascular ECM accumulation and atherosclerosis.

\section{Methods}

Antibodies, reagents and plasmids Rabbit antibody, raised to GST fusion protein of the C-terminal fragment of mouse CDA1, was affinity-purified using a method described previously [12]. Antibodies to phospho-SMAD3 (Ser433/ 435) (\#9514) and to SMAD2/3 (sc-8332) were from Cell Signaling Technology (Beverly, MA, USA) and Santa Cruz Biotechnology (Santa Cruz, CA, USA), respectively. The antibody to $\alpha$-tubulin (T5168) was from Sigma-Aldrich (St Louis, MO, USA) and recombinant human TGF- $\beta 1$ from United States Biological Inc. (Swampscott, MA, USA). Constructs of p3TP-Lux and $\mathrm{p}(\mathrm{CAGA})_{12}$-Luc have been described previously $[28,29]$.

Cell culture Mouse VSMCs isolated from mouse aorta have been described previously [30]. The cells were cultured in DMEM/F12 plus $10 \%$ (vol./vol.) fetal bovine serum at $37^{\circ} \mathrm{C}$ with $5 \% \mathrm{CO}_{2}$. VSMCs were stably transduced by vector control retrovirus or retrovirus expressing siRNA m957 silencing the CDA1-encoding gene Tspyl2 as described previously for the human CDA1-encoding gene TSPYL2 in HeLa cells [13]. Tspyl2 siRNA m957 targets a region of the open reading frame conserved in human and mouse.

Adenovirus constructs Adenovirus constructs to overexpress mouse CDA1-encoding gene Tspyl2 or to express hairpin-structured siRNA to silence Tspyl2 were prepared using a vector system (AdEasy; Quantum Biotechnologies, Montreal, QC, Canada). Briefly, the DNA fragment encoding Myc-tagged mouse CDA1 was cloned into the transfer vector, pAdTrack-CMV. The expression cascade containing $\mathrm{H} 1$ promoter and the DNA fragment coding for the hairpin structured Tspyl2 siRNA m957 was cloned into the transfer vector, pAdTrack. The linearised transfer vector constructs were co-transformed with pAdEasy-1 plasmid into $E$. coli strain BJ5183 allowing recombination to generate a recombinant adenovirus plasmid, which was then used to produce infectious, but replication-defective adenoviruses in HEK293 packaging cells. Viral particles were purified by double caesium chloride gradient ultra-centrifugations.

Initially, the HEK293 packaging cells failed to produce CDA1-producing adenovirus, probably due to overproduction of CDA1 protein, which inhibits DNA synthesis and arrests cell proliferation, thereby adversely affecting ade- 
novirus production $[12,13]$. To overcome this problem, HEK293 packaging cells were stably transduced with the retrovirus expressing Tspyl2 siRNA m957 [13], which significantly reduced mRNA and protein levels of CDA1 even when used to produce CDA1-producing adenoviruses.

Gene-specific mRNA quantitation by real-time RT-PCR Genespecific mRNA levels were quantitatively determined as described [13] using a PCR system (7500 Fast RealTime PCR; Applied Biosystems, Foster City, CA, USA) and normalised against endogenous $18 \mathrm{~S}$ ribosome RNA using ribosomal RNA control reagents (TaqMan: Applied Biosystems). Results are shown as fold change (arbitrary unit) relative to control. Probes and primers used to determine gene-specific mRNA levels are listed in Electronic supplementary material (ESM) Table 1.

Luciferase reporter assay The luciferase reporter assay has been described previously [13]. Briefly, mouse VSMCs stably transduced with vector control retrovirus (pSUPERretro-puromycin) or retrovirus expressing $T_{s p y l 2}$ siRNA m957 were co-transfected by electroporation with pGL3BASIC (Promega, Madison, WI, USA), p3TP-Lux or $\mathrm{p}(\mathrm{CAGA})_{12}$-Luc $(15 \mu \mathrm{g})$ and CMV- $\beta$-galactosidase $(3 \mu \mathrm{g})$ plasmids. Complete medium was replaced with serum-free medium $24 \mathrm{~h}$ after electroporation. TGF- $\beta$ at final concentration of $1.25 \mathrm{ng} / \mathrm{ml}$ or no TGF- $\beta$ was added to treat cells for a further $24 \mathrm{~h}$. Luciferase and $\beta$-galactosidase activities of each sample were analysed using assay systems (Luciferase Assay, catalogue number E1501; $\beta$-Galactosidase Enzyme Assay, catalogue number E2000; Promega).

Animal model The diabetic Apoe $e^{-/}$mouse is an extensively characterised model of diabetes-associated atherosclerosis, as described previously [4, 31, 32]. Briefly, 6-week-old Apoe ${ }^{-/}$male mice on a C57BL6 background were rendered diabetic by five daily intraperitoneal injections of streptozotocin (Boehringer-Mannheim, Mannheim, Germany) at a dose of $55 \mathrm{mg} / \mathrm{kg}$ in citrate buffer. Non-diabetic Apoe - $^{--}$ control animals were injected with buffer alone. Only animals injected with streptozotocin and with blood glucose levels $>15 \mathrm{mmol} / \mathrm{l}$ at 1 week after injection were considered diabetic. All animals were housed on a $12 \mathrm{~h} \mathrm{light/dark} \mathrm{cycle}$ with free access to water and standard mouse chow (Specialty Feeds, WA, Australia). The animal studies were approved by the AMREP Animal Ethic Committee according to principles in line with international guidelines including the 'Principles of laboratory animal care' (National Institutes of Health, 1985). Animals were killed 10 and 20 weeks after induction of diabetes. Body weight and blood pressure (systolic BP) were measured prior to killing. Methods for the measurement of systolic BP by tail cuff plethysmography, $\mathrm{HbA}_{1 \mathrm{c}}$, plasma lipid and glucose levels, as well as en face analysis of aortic plaque area (\% intimal area stained with Sudan IV) have been described previously $[33,34]$. Whole aortas were cleaned by removing fat and adventitia, and then used for isolation of total RNA or for en face analysis of aortic plaque area. Cleaned aortic tissues were also used for preparation of paraffin sections as described previously $[4,35,36]$.

Assessment of plaque area The en face analysis of atherosclerotic lesions was conducted as described previously [4]. The plaque area was calculated as the proportion (\%) of aortic intimal surface area occupied by red stained plaques (Adobe Photoshop 6.0.1; Adobe Systems, San Jose, CA, USA).

Immunohistochemical and Trichrome staining Immunohistochemical staining for various proteins in the paraffin sections was carried out as described previously [4]. Briefly, after incubation with primary antibodies (1:2,000 dilution for CDA1, 1:200 for connective tissue growth factor [CTGF]), biotinylated horse anti-rabbit or anti-mouse immunoglobulin (Vector Laboratories, Burlingame, CA, USA) was used as a second antibody, then followed by horseradish peroxidaseconjugated streptavidin. Peroxidase activity was identified by reaction with 3,3'-diaminobenzidine tetrahydrochloride (Sigma Chemical, St Louis, MO, USA). Collagen content was shown by Masson's Trichrome staining method [4].

\section{Results}

Phenotypic data of Apoe ${ }^{-/}$mice with or without diabetes for 20 weeks As summarised in Table 1, the Apoe ${ }^{-/-}$animals after 20 weeks of diabetes had significantly higher plasma glucose concentrations and higher $\mathrm{HbA}_{1 \mathrm{C}}$ levels than nondiabetic $A p o e^{-/-}$mice. The body weight of the diabetic mice was significantly lower and plasma total cholesterol, HDL and LDL were higher than in control mice (Table 1). Diabetic Apoe $^{-/-}$mice did not show a significant change in plasma triacylglycerol or systolic blood pressure when compared with the non-diabetic group (Table 1).

Diabetes accelerates development of atherosclerosis in Apoe $^{-/}$mice After 20 weeks of diabetes mice had increased levels of atherosclerosis compared with age matched non-diabetic Apoe ${ }^{-/}$mice (Fig. 1). Increased staining of plaques in the arch, thoracic and abdominal aorta of diabetic mice was observed (Fig. 1). In diabetic mice, the arch region of the aorta had a higher percentage plaque area than the thoracic and abdominal aortic regions (Fig. 1).

Concomitant increases in expression levels of Tspyl2 and profibrotic growth factors in atherosclerotic aortas from 
Table 1 Metabolic and blood pressure data for $\mathrm{Apoe}^{-/}$mice

\begin{tabular}{lcc}
\hline Variable & $\begin{array}{l}\text { Non-diabetic } \\
(n=9-20)\end{array}$ & $\begin{array}{l}\text { Diabetic } \\
(n=9-20)\end{array}$ \\
\hline Plasma glucose (mmol/l) & $12.0 \pm 0.5$ & $30.2 \pm 1.2^{\mathrm{a}}$ \\
HbA $_{1 \mathrm{c}}(\%)$ & $4.3 \pm 0.1$ & $14.4 \pm 0.3^{\mathrm{a}}$ \\
Total cholesterol (mmol/l) & $12.2 \pm 0.6$ & $18.8 \pm 1.0^{\mathrm{a}}$ \\
HDL (mmol/l) & $2.6 \pm 0.2$ & $3.5 \pm 0.2^{\mathrm{b}}$ \\
LDL (mmol/l) & $8.5 \pm 0.4$ & $14.0 \pm 0.9^{\mathrm{a}}$ \\
Body weight $(\mathrm{g})$ & $32.7 \pm 0.5$ & $24.4 \pm 0.5^{\mathrm{a}}$ \\
Triacylglycerol $(\mathrm{mmol} / \mathrm{l})$ & $2.4 \pm 0.1$ & $2.8 \pm 0.2$ \\
Blood pressure $(\mathrm{mmHg})$ & $106 \pm 3$ & $110 \pm 5$ \\
\hline
\end{tabular}

Data are shown as mean \pm SEM

${ }^{\mathrm{a}} p<0.001 ;{ }^{\mathrm{b}} p=0.02$ vs non-diabetic $A p o e^{-/-}$mice

diabetic Apoe $e^{--}$mice We found that mRNA levels of CDA1-encoding gene Tspyl2 as well as of the profibrotic growth factors $\operatorname{Tgf}-\beta$ and $C \operatorname{tg} f$ increased 1.5 to twofold in aortas of $\mathrm{Apoe}^{-/-}$mice with 10 weeks of diabetes (Fig. 2a). Expression of these genes increased further, namely by more than fivefold (Tspyl2), tenfold (Tgf- $\beta$ ) and more than 15-fold (Ctgf), respectively, at 20 weeks of diabetes when compared with age-matched non-diabetic animals (Fig. 2b).

CDA1 protein was strongly stained in vessels from mice after 20 weeks of diabetes, being present extensively in the atherosclerotic plaques (Fig. 2c). In control $\mathrm{Apoe}^{-/}$mice

\section{a}

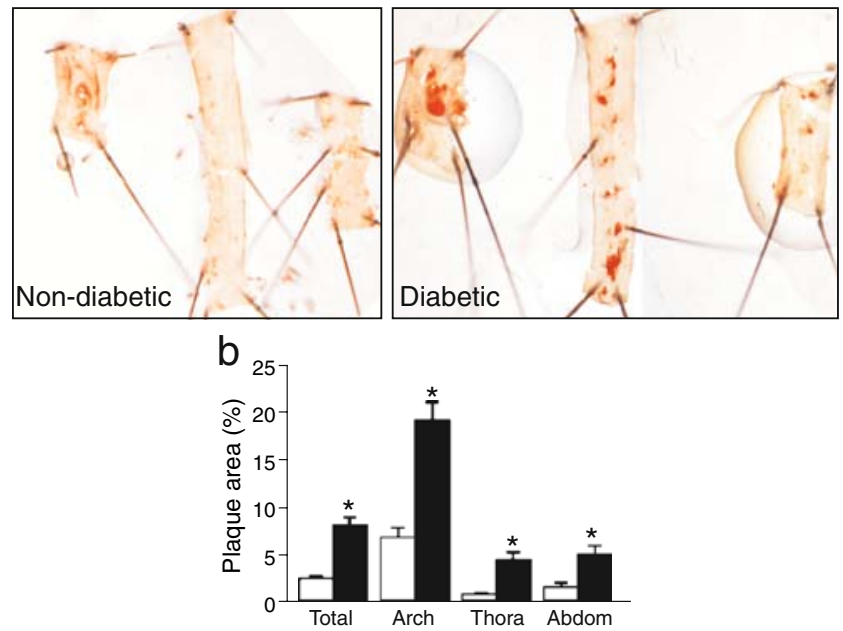

Fig. 1 Diabetes accelerates development of atherosclerosis in $A p o e^{-1-}$ mice. a Representative view of atherosclerotic plaques stained by Sudan IV (red) in arch, thoracic and abdominal (left to right) aortas from 20-week non-diabetic and diabetic $\mathrm{Apoe}^{-/-}$mice. b Analysis of the quantified plaque areas in total, arch, thoracic (Thora) and abdominal (Abdom) aortas from 20-week non-diabetic group (white bar, $n=10$ ) and diabetic group (black bar, $n=8$ ) of Apoe ${ }^{-/-}$mice. Values are means \pm SE. SE $(n=8-10)$ is shown as error bar. ${ }^{*} p<0.001$ vs non-diabetic group
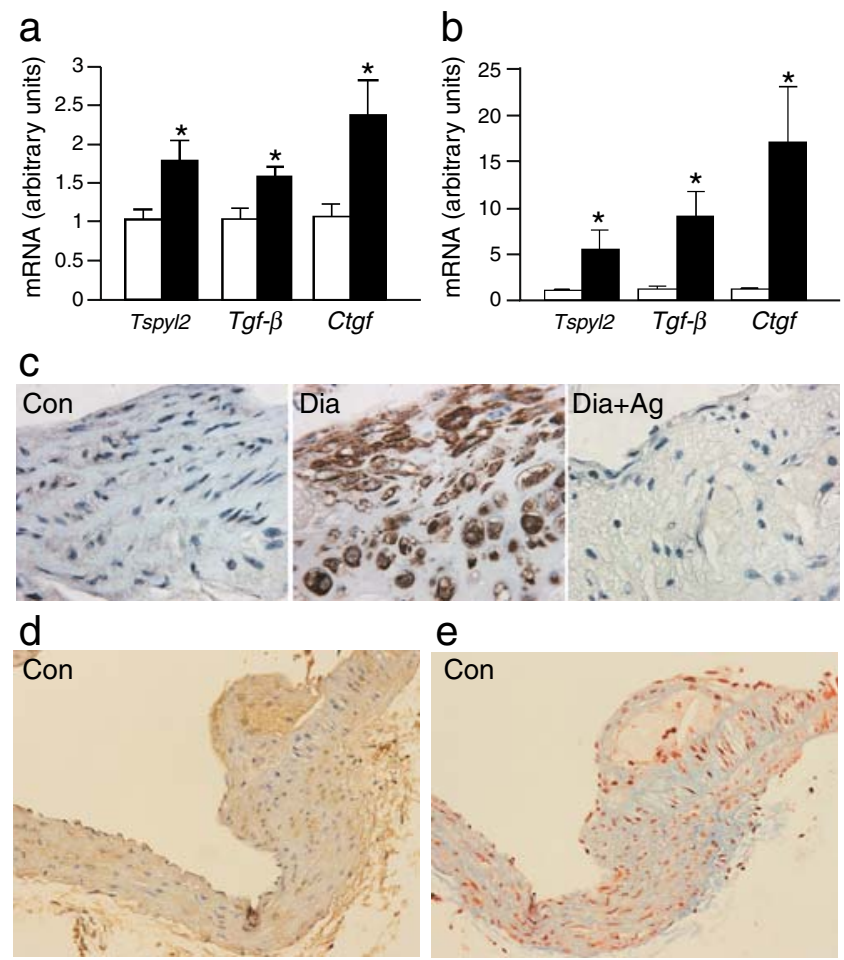

e
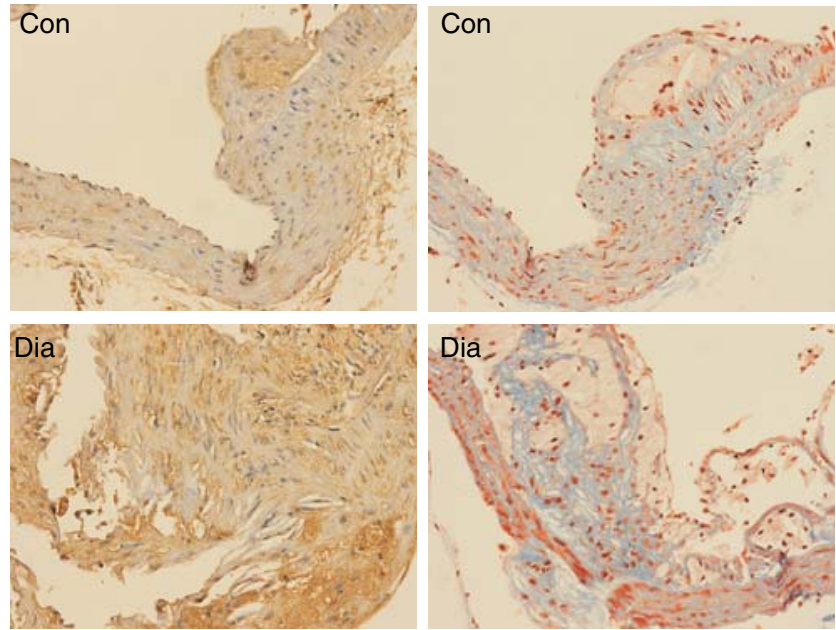

Fig. 2 Aortic Tspyl2 expression is increased in diabetic Apoe $e^{-/}$mice. a Relative mRNA levels of Tspyl2, Tgf- $\beta$ and $C \operatorname{tg} f$ as determined by quantitative real-time PCR in aorta of 10-week-old or (b) 20-week-old diabetic (black bars) and non-diabetic (white bars) Apoe $^{-1}$ mice. Values are means $\pm \mathrm{SE}$. SE $(n=5-7)$ is shown as error bar. ${ }^{*} p<0.05$ vs non-diabetic group. $\mathbf{c}$ Immunohistochemical staining of CDA1 in 20week diabetic (Dia) and non-diabetic control (Con) $\mathrm{Apoe}^{-/}$mouse aorta sections. The diabetic aorta section was also stained with the same anti-CDA1 antibody that was pre-absorbed with CDA1 antigen $(\mathrm{Dia}+\mathrm{Ag})$. d Immunohistochemical staining for CTGF and (e) Trichrome staining for ECM in 20-week non-diabetic (Con) and diabetic (Dia) aorta sections

there was much less atherosclerosis [4] and very little staining of CDA1 (Fig. 2c). Staining of CDA1 in the diabetic atherosclerotic plaque was removed by preabsorption of the antibody with CDA1 antigen, confirming the specificity of CDA1 staining (Fig. 2c).

CTGF protein levels have been previously characterised in detail and shown to be elevated in this animal model [4]. Our immunohistochemical staining for CTGF showed a similar result, with higher levels of CTGF in the diabetesaccelerated atherosclerotic plaque than in non-diabetic controls (Fig. 2d). This is consistent with a role for CDA1 in promoting gene expression of profibrotic Ctgf in vivo 
and is consistent with our in vitro finding that CDA1 is involved in TGF- $\beta$ signalling and is able to promote expression of Ctgf.

Enhanced accumulation of ECM in diabetic atherosclerotic aortas of $\mathrm{Apoe}^{-/}$mice Associated with upregulation of CDA1 and TGF- $\beta$, the diabetic Apoe $e^{--}$atherosclerosis model displayed increased accumulation of ECM in the plaque (Fig. 2e). This result is consistent with our hypothesis that CDA1 has a role in enhancing TGF- $\beta$ signalling leading to upregulation of CTGF and ultimately leading to increased production and accumulation of vascular ECM in vivo.

CDA1 knockdown abrogates TGF- $\beta$-stimulated SMAD3 activation in VSMCs In order to establish a pathological role for overexpression of CDA1-encoding gene Tspyl2 leading to vascular ECM accumulation, we examined whether CDA1 plays a role in TGF- $\beta$-stimulated SMAD phosphorylation and transcriptional activities. We stably transduced VSMCs with either a vector control retrovirus or a retrovirus expressing hairpin-structured siRNA m957 [13] to reduce the CDA1 level. The cells were treated with TGF- $\beta$ at specified concentrations for $30 \mathrm{~min}$. CDA1 was undetectable in mouse VSMCs with its gene being silenced by Tspyl2 siRNA m957, whereas CDA1 protein levels in control VSMCs were increased by TGF- $\beta$ treatment for $30 \mathrm{~min}$ (Fig. 3a). SMAD3 phosphorylation was undetectable in cells without TGF- $\beta$ treatment or with $0.1 \mathrm{ng} / \mathrm{ml}$ TGF- $\beta$. In contrast, SMAD3 phosphorylation was markedly increased with $0.5 \mathrm{ng} / \mathrm{ml} \mathrm{TGF}-\beta$ and further increased with $1.25 \mathrm{ng} / \mathrm{ml}$ in a dose-dependent manner. SMAD3 phosphorylation stimulated by TGF- $\beta$ at the same concentrations was markedly attenuated by the decrease in the CDA1 level due to Tspyl2 siRNA knockdown, whereas total SMAD2/3 levels were not reduced (Fig. 3a). Attenuation of TGF- $\beta$-stimulated SMAD3 phosphorylation by Tspyl2 knockdown was also observed with TGF- $\beta$ treatment at higher concentrations (Fig. 3a). These results demonstrate that CDA1 participates in TGF- $\beta$ signalling and plays a critical role in amplifying the stimulation by TGF- $\beta$ of SMAD3 phosphorylation; they also show that CDA1 protein levels play a pivotal role in modulating SMAD phosphorylation and activation in response to TGF- $\beta$.

The role for CDA1 in mediating signalling and transcriptional activities of TGF- $\beta$ was examined in control and Tspyl2 knockdown VSMCs by measuring luciferase activities of a TGF- $\beta$-responsive luciferase reporter construct, p3TP-Lux, and a SMAD3-specific luciferase reporter construct, p(CAGA) $)_{12}$-Luc (Fig. 3b). Background levels of luciferase activities for the promoterless luciferase gene construct pGL3-BASIC in either vector retrovirus- or Tspyl2 siRNA retrovirus-transduced VSMCs were not
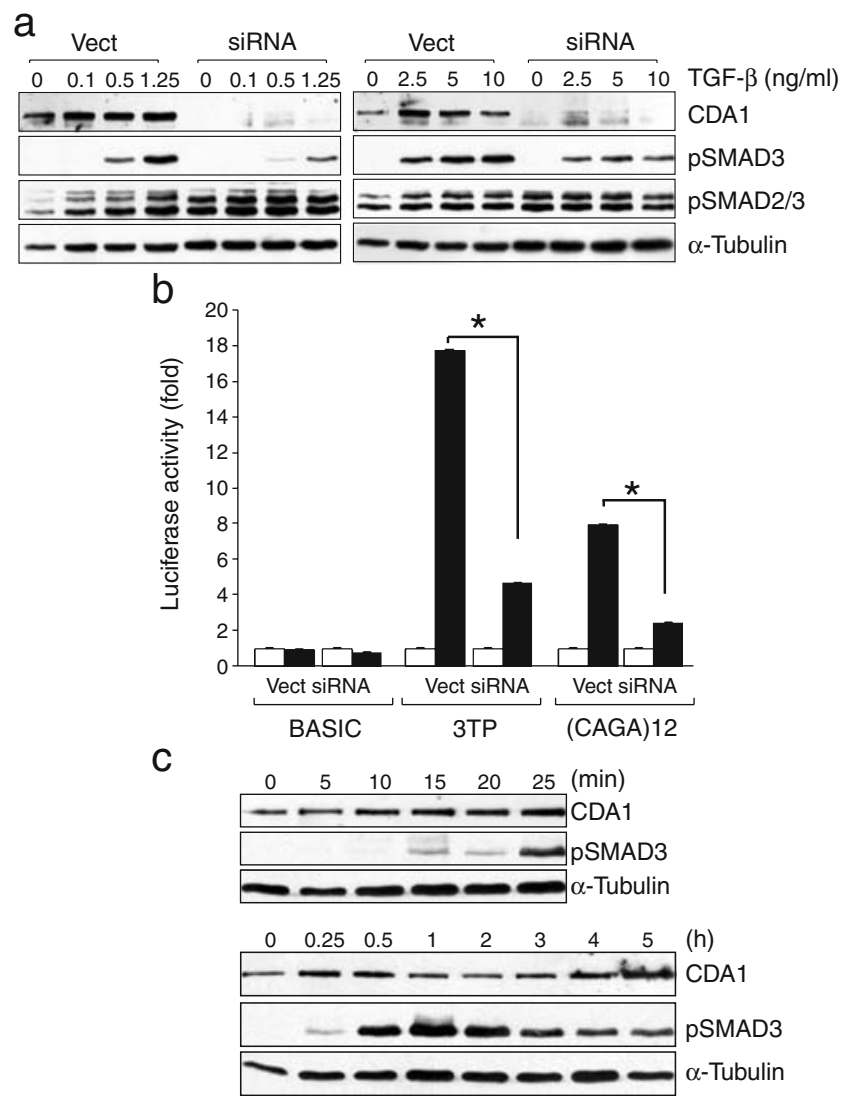

Fig. $3 \mathrm{CDA} 1$ is involved in TGF- $\beta$ signalling. a Western blot showing CDA1, phosphor-SMAD3, total SMAD2/3 and $\alpha$-tubulin in control (Vect) and CDA1-encoding gene Tspyl2 siRNA (siRNA) knockdown VSMCs treated with TGF- $\beta$ at specified concentrations for $30 \mathrm{~min}$. b Luciferase activities of TGF- $\beta$-responsive reporter constructs (3TP, CAGA) and a control (BASIC) in VSMCs treated or not treated with TGF- $\beta$ at $2.5 \mathrm{ng} / \mathrm{ml}$ for $24 \mathrm{~h}$. Values are means \pm SE. SE $(n=6)$ is shown as error bar. ${ }^{*} p<0.001$. $\mathbf{c}$ Western blot of CDA1, phosphor-SMAD 3 and $\alpha$-tubulin in VSMCs treated with TGF- $\beta$ at $1 \mathrm{ng} / \mathrm{ml}$ for specified times

changed by TGF- $\beta$ treatment (Fig. 3b). p3TP-Lux activity was stimulated $>17$-fold by TGF- $\beta$ treatment at $2.5 \mathrm{ng} / \mathrm{ml}$ for $24 \mathrm{~h}$ in vector control cells, whereas CDA1 knockdown attenuated the stimulatory effect of TGF- $\beta$ by $>70 \%$ (Fig. 3b). Similarly, TGF- $\beta$ stimulated an eightfold increase in luciferase activity of the $\mathrm{p}(\mathrm{CAGA})_{12}$-Luc in vector control cells, but this increase was attenuated by more than $60 \%$ in the Tspyl2 siRNA knockdown cells (Fig. 3b). These results indicate a role for CDA1 in modulating TGF- $\beta$ signalling, leading to regulation of TGF- $\beta$-responsive gene promoters, a process involving SMAD3.

$C D A 1$ protein levels rapidly increase in response to $T G F-\beta$ treatment To further confirm the response of CDA1 with increase in CDA1 protein levels to the TGF- $\beta$ treatment, as observed in Fig. 3a, we treated VSMCs with TGF- $\beta$ for various periods of time. TGF- $\beta$ rapidly increased CDA1 
protein levels after $10 \mathrm{~min}$ and these progressively increased up to $25 \mathrm{~min}$ (Fig. 3c). Following the rise in CDA1, phosphorylated SMAD3, detected by an antibody to phospho-SMAD3 (Ser433/435), rose to a detectable level after 15 and $20 \mathrm{~min}$, and was markedly increased at $25 \mathrm{~min}$ (Fig. 3c). In a longer time-course study, the rapid induction of CDA1 and SMAD3 phosphorylation by TGF- $\beta$ was confirmed at 15 and $30 \mathrm{~min}$ (Fig. 3c). Furthermore, CDA1 levels dropped from the peak to the control level after 1 to $2 \mathrm{~h}$ of TGF- $\beta$ treatment, gradually rising again after $3 \mathrm{~h}$ and reaching a second peak after $5 \mathrm{~h}$. SMAD3 phosphorylation peaked at $1 \mathrm{~h}$ and then gradually decreased. These findings confirm that TGF- $\beta$ is able to rapidly increase CDA1 protein levels, which further amplifies the ability of TGF- $\beta$ to stimulate SMAD3 phosphorylation and activities (Fig. 3a, b).

Physiological role for $C D A 1$ in regulating $C t g f$ and ECM genes We examined the effect of knockdown of CDA1-encoding gene Tspyl2 on TGF- $\beta$ target genes, including $C t g f$ and ECM genes in VSMCs. VSMCs were un-transduced or stably transduced with a vector control retrovirus or with a retrovirus expressing hairpin-structured siRNA targeting a human sequence that is not conserved in mouse genome. This retrovirus expressing hairpinstructured siRNA was for use as an irrelevant siRNA control. The retrovirus expressing Tspyl2 siRNA m957 [13] is described in Fig. 3. Tspyl2 siRNA reduced Tspyl2 mRNA levels by $\sim 40 \%$ in VSMCs (Fig. 4). Expression of Ctgf, collagen I, III and IV, and fibronectin was significantly decreased in Tspyl2 siRNA knockdown cells by $20 \%$ to $80 \%$ (Fig. 4). These results suggest a physiological role for endogenous CDA1 in regulating TGF- $\beta$ target genes, including Ctg $f$ and ECM genes, in VSMCs, consistent with its role in TGF- $\beta$ signalling and transcriptional regulation of TGF- $\beta$-responsive gene promoters (Fig. 3a, b).

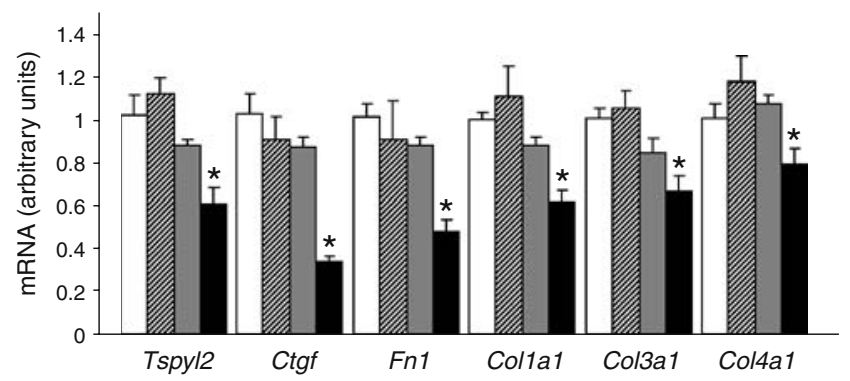

Fig. 4 Knockdown of CDA1-encoding gene Tspyl2 results in decreased mRNA levels of Ctgf and various ECM genes. mRNA levels for Tspyl2, Ctgf, Fn1 and Colla1, Col3al and Col4al in VSMCs transduced with no virus (white bars), vector retrovirus (hatched bars), retrovirus expressing an irrelevant siRNA (grey bars) or retrovirus expressing siRNA silencing CDA1 (black bars). Values are means \pm SE. SE $(n=6)$ is shown as error bar. $* p<0.001$ vs VSMC group with no virus
$C D A 1$ is required for $T G F-\beta$ to stimulate expression of Ctgf and ECM genes We used adenovirus constructs to either overexpress or knockdown CDA1-encoding gene Tspyl2 in VSMCs. Uninfected VSMCs and VSMCs infected with a vector adenovirus were used as controls (Fig. 5). After $24 \mathrm{~h}$ of
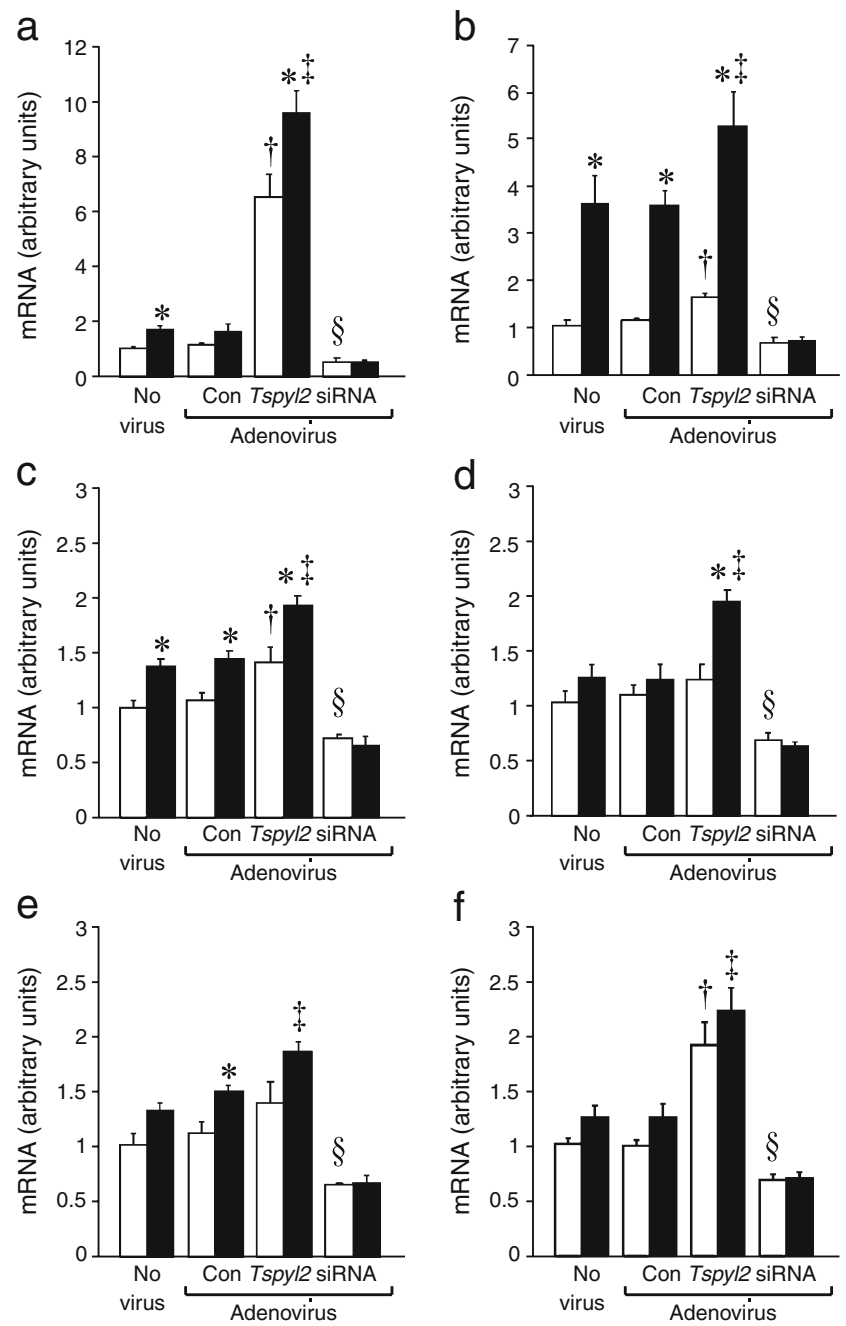

Fig. 5 CDA1 is critical for TGF- $\beta$ to regulate expression of Ctgf and various ECM genes. Mouse VSMCs were infected with no virus, a vector adenovirus (Con adenovirus), an adenovirus expressing mouse CDA1-encoding gene Tspyl2 (Tspyl2 adenovirus) or an adenovirus expressing siRNA m957 to silence Tspyl2 (siRNA adenovirus). The same quantity of adenoviruses $\left(\sim 1 \times 10^{9}\right.$ plaque forming unit) was added to $\sim 5 \times 10^{5}$ VSMCs for $24 \mathrm{~h}$ to achieve the same levels of enhanced green fluorescent protein as assessed under a fluorescent microscope. After removal of the medium containing the adenoviruses, serum-free DMEM/F12 medium with or without TGF- $\beta$ at $1.25 \mathrm{ng} / \mathrm{ml}$ was added and the cells were treated for a further $24 \mathrm{~h}$. mRNA levels for CDA1encoding gene Tspyl2 (a), Ctgf (b), and genes encoding for collagen types I (Collal) (c), III (Col31a) (d) and IV (Col4al) (e), and fibronectin $(F n l)($ f) were determined by real-time RT-PCR and are shown as arbitrary units. Values are means \pm SE. SE $(n=6)$ is shown as error bar. ${ }^{*} p<0.05$ vs untreated group; ${ }^{\dagger} p<0.05$ vs untreated group of no virus cells; ${ }^{\star} p<0.05$ vs no virus and control adenovirus in the presence of TGF- $\beta ;{ }^{\S} p<0.05$ vs no virus (white bar) and control adenovirus (Con adenovirus, white bar) in the absence of TGF- $\beta$ treatment 
adenovirus infection, the cells were treated with TGF- $\beta$ for a further $24 \mathrm{~h}$. As shown in Fig. 5, the Tspyl2-overexpressing adenovirus increased Tspyl2 mRNA levels by more than sixfold whereas the siRNA adenovirus reduced Tspyl2 mRNA levels by $>40 \%$. TGF- $\beta$ treatment moderately increased endogenous Tspyl 2 mRNA levels in the control groups as well as in Tspyl2-adenovirus-infected VSMC group, but this was not observed in the siRNA adenovirus-infected VSMCs. TGF- $\beta$ stimulated $\operatorname{Ctg} f$ mRNA levels more than threefold in the two control groups. Overexpression of CDA1-encoding gene Tspyl2 also increased Ctgf mRNA levels by $>70 \%$. TGF- $\beta$ treatment in the Tspyl2-adenovirus-infected VSMC was associated with an even greater increase in Ctgf expression of $>500 \%$. TGF- $\beta$ failed to stimulate $C \operatorname{tg} f$ expression when CDA1-encoding gene Tspyl2 was silenced by the siRNA-adenovirus in VSMCs (Fig. 5). In a similar pattern to that seen with Ctgf, ECM genes including collagen types I, III and IV, and fibronectin were stimulated moderately by TGF- $\beta$ or CDA1 alone, and were stimulated to a greater extent by the combination of TGF- $\beta$ and CDA1.

In the presence of TGF- $\beta$, overexpression of the CDA1encoding gene $T_{s p y l}$ significantly increased mRNA levels of $C \operatorname{tg} f$ and various ECM genes compared with the TGF- $\beta$ treatment groups with either no virus or with the control adenovirus. This demonstrates the ability of CDA1 to enhance the effect of TGF- $\beta$ in upregulating these target genes. Furthermore, silencing of CDA1-encoding gene Tspyl2 not only reduced basal mRNA levels of Ctgf and ECM genes, consistent with the results seen with the retrovirus as shown in Fig. 4, but also prevented TGF- $\beta$ from stimulating these genes (Fig. 5). The effect of Tspyl2 knockdown on TGF- $\beta$-stimulated gene expression is consistent with the Tspyl2 knockdown-induced attenuation of TGF- $\beta$ signalling (Fig. 3a, b).

CDA1 increases expression levels of Tgf- $\beta$ and Tgf- $\beta$ receptor type I, but not type II To examine the mechanism whereby CDA 1 modulates the TGF- $\beta$ signalling pathway leading to regulation of TGF- $\beta$ target genes such as $C \operatorname{tg} f$ and various ECM genes, we determined the effects of Tspyl2 knockdown and overexpression on expression of $T g f-\beta$ and its receptors. We found that Tspyl 2 knockdown, as described in Fig. 4, resulted in significant decreases in mRNA levels of $T g f-\beta$ by $\sim 30 \%$ and of the TGF- $\beta$ receptor type I or $T \beta r I$ (also known as $T g f b r I$ ) by $\sim 50 \%$, but not of the TGF- $\beta$ receptor type II or $T \beta r I I$ (also known as $T g f b r 2$; Fig. 6a). In contrast, adenovirus-delivered CDA1 overproduction resulted in increase of $T g f-\beta$ by $\sim 150 \%$ and of the $T \beta r I$ by $\sim 300 \%$, but not of the TRrII (Fig. $6 \mathrm{~b}$ ). These results show that CDA1 is able to increase the expression levels of the ligand and receptor levels of the TGF- $\beta$ signalling pathway, providing a mechanism to explain the action of CDA1 in mediating the profibrotic effect of TGF- $\beta$.
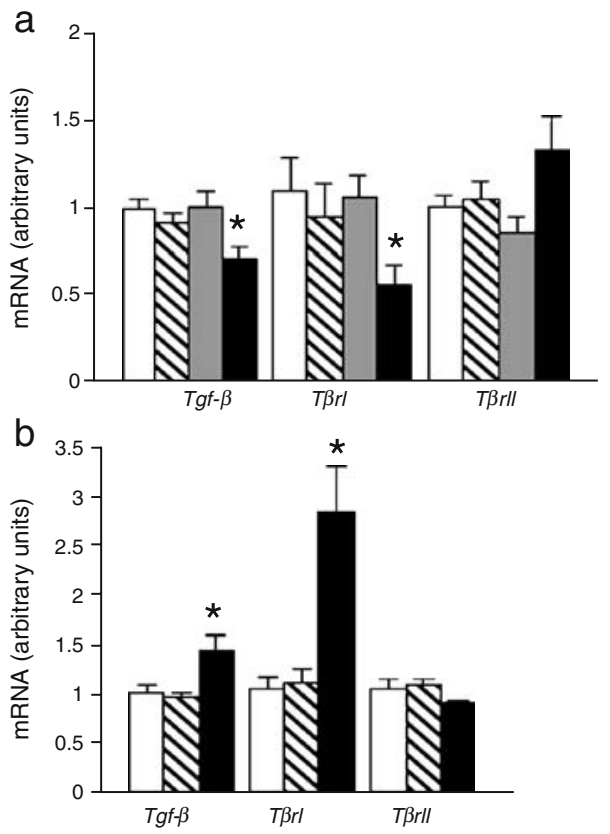

Fig. 6 CDA1 increases expression of Tgf- $\beta$ and $T \beta r I$, but not $T \beta r I I$. The mRNA levels of the above are shown in the same VSMCs with (a) CDA1 knockdown and controls as described for Fig. 4 (key identical) or with (b) overexpression of CDA1-encoding gene Tspyl2 as described in Fig. 5, but without TGF- $\beta$ treatment, including no virus (white bars), vector (hatched bars) and CDA1-producing adenovirus (black bars) infected cells. Values are means \pm SE. SE $(n=6)$ is shown as error bar. ${ }^{*} p<0.001$ vs no virus group

\section{Discussion}

This study used a well characterised animal model of diabetesaccelerated atherosclerosis, the streptozotocin induced diabetic $A p o e^{-/}$mouse, which develops aortic plaques with pathological features relevant to human disease [32]. As characterised in detail previously $[4,5]$, this model has increased vascular ECM accumulation, which is associated with accelerated vascular disease, manifested as rapid development of atherosclerotic plaques. ECM remodelling in blood vessels is an early event in response to pathological insults, with vascular fibrosis likely to further contribute to the subsequent development of atherosclerosis. Specifically, ECM accumulation may help increase plaque size, leading to narrowing of the lumen of these vessels.

The present study reports a novel observation that expression of Tspyl2, the gene encoding for CDA1, is increased in the atherosclerotic aortas of diabetic Apoe - $^{--}$ mice. To further explore the potential biological role of CDA1, we carried out a series of in vitro experiments increasing cellular levels of CDA1 in mouse VSMCs to mimic the in vivo diabetes context in order to demonstrate the profibrotic effect of CDA1. Our siRNA data specifically show the efficacy of targeting CDA1 in attenuating TGF- $\beta$ stimulated expression of ECM genes. 
Expression levels of CDA1-encoding gene Tspyl2 were increased progressively in the aorta of $A p o e^{-/}$mice after 10 to 20 weeks of diabetes, accompanied by the progressive development of atherosclerosis and increased levels of TGF- $\beta$, ECM content and atherosclerotic plaque area (Figs 1 and 2), as previously reported [4, 35]. This suggests that regulatory molecules such as CDA1 are induced at an earlier stage, before the disease has become manifest. These findings are therefore consistent with a role for CDA1 in the development and progression of atherosclerosis in these mice, particularly in the diabetic context.

The hypothesis that an increase in CDA1-encoding gene Tspyl2 expression in diabetic atherosclerosis is due to an enhanced level of TGF- $\beta$ and plays a pathological role by enhancing the profibrotic effect of TGF- $\beta$ was investigated in a series of in vitro studies involving overexpression and inhibition of vascular $T_{s p y l 2}$ expression. Specifically, CDA1 protein levels were rapidly increased by TGF- $\beta$ treatment, followed by SMAD3 phosphorylation. CDA1 protein levels gradually decreased for 1 to $2 \mathrm{~h}$ after the peak level, rising again at 4 to $5 \mathrm{~h}$ after TGF- $\beta$ treatment. The first increase in CDA1 is apparently a result of exogenous TGF- $\beta$ stimulation. The second peak in CDA1 at 4 to $5 \mathrm{~h}$ may reflect an autocrine effect of endogenous TGF- $\beta$, which is consistent with a previous observation that the protein level of CDA1 is increased by TGF- $\beta$ treatment at 4 and $8 \mathrm{~h}$ in a human lung cell line [23]. The ability of CDA1 to promote expression of $C \operatorname{tg} f$ and the ECM genes, including collagen I, III and IV, and fibronectin, in VSMCs was demonstrated by the increase in expression of these genes following CDA1 overproduction.

The critical roles for CDA1 in mediating TGF- $\beta$ signalling and TGF- $\beta$-dependent stimulation of expression of $C \operatorname{tg} f$ and various ECM genes were clearly demonstrated using an siRNA CDA1-encoding gene knockdown approach. Specifically, Tspyl2 knockdown led to marked attenuation of TGF- $\beta$ signalling, as well as abrogation of the profibrotic effect of TGF- $\beta$. These data not only show that CDA1 has a pathological role in promoting the development of atherosclerosis in diabetes, but also support the potential efficacy of targeting CDA1 in order to inhibit or reduce ECM production as a result of enhanced TGF- $\beta$ action coordinated by elevated production of CDA1.

Unlike directly targeting TGF- $\beta$ or TGF- $\beta$ receptors, which results in deleterious effects due to inhibition of other important biological effects of TGF- $\beta$, targeting CDA1 specifically appears to block only the enhanced action of TGF- $\beta$. Knockdown of the CDA1-encoding gene Tspyl2 markedly attenuated, but did not completely block TGF- $\beta$-stimulated SMAD3 phosphorylation and transcriptional activities (Fig. 3). However, this effect appears to be sufficient to prevent the stimulatory effect of TGF- $\beta$ on its target genes associated with ECM production, including Ctgf, collagen I, III and IV, and fibronectin (Fig. 5). Based on these results, it is suggested that targeting CDA1 will be effective in inhibiting ECM production increased by the enhanced profibrotic action of TGF- $\beta$. Furthermore, it is predicted that targeting CDA1 may not affect key physiological roles of TGF- $\beta$, since SMAD phosphorylation and transcriptional activities of TGF- $\beta$ were not completely blocked (Fig. 3).

The molecular pathway whereby CDA1 mediates the profibrotic effect of TGF- $\beta$ and angiotensin II involves SMAD3, a key TGF- $\beta$ signalling molecule involved in vascular fibrosis [37] and diabetic nephropathy [38, 39]. The effects of CDA1 reduction and overproduction on Tgf- $\beta$ and $T \beta r I$ gene expression provide a molecular mechanism to explain the role of CDA1 in TGF- $\beta$ signalling and target gene expression. The TGF- $\beta$ receptor type I has dual specificity kinase activities directly responsible for phosphorylating R-SMAD proteins including SMAD3 [40, 41] and activating the ERK MAPK pathway [21]. This is consistent with a role for CDA1, not only in enhancing TGF- $\beta$-stimulated SMAD3 activity, but also in activating ERK MAPK as has been shown previously [13]. Indeed, the ERK MAPK pathway has been previously reported to be involved in diabetic complications [42] and the crosstalk between the SMAD and MAPK pathways is considered to play an important role in diabetic tissue scarring in the vasculature and kidney [43].

In summary, CDA1 is implicated in diabetes-induced vascular ECM accumulation by enhancing TGF- $\beta$ signalling, leading to increased gene expression of Ctgf and ECM proteins. TGF- $\beta$ is upregulated in diabetes with enhanced TGF- $\beta$ signalling and excess accumulation of vascular ECM, as seen in diabetes-accelerated atherosclerosis. Indeed, increased TGF- $\beta$ activity induces CDA1, with this molecule appearing to be critical for the profibrotic effect of TGF- $\beta$. Targeting CDA1 is effective at attenuating the profibrotic action of TGF- $\beta$, but does not completely block TGF- $\beta$ signalling. Therefore, CDA1 appears to be an attractive molecular target for treating vascular fibrosis, atherosclerosis and other vascular disorders associated with enhanced TGF- $\beta$ action and tissue scarring as commonly seen in diabetes.

Acknowledgements This work was funded by the National Health and Medical Research Council of Australia, the National Heart Foundation of Australia and the Juvenile Diabetes Research Foundation.

Duality of interest Z. Chai, Z. Cao and M. E Cooper have an international patent at National Phase with the Baker IDI Heart and Diabetes Institute and Dia-B Tech, Melbourne, Australia: 'Methods and compositions for treating disorders of the extracellular matrix'. Otherwise there is no duality of interest associated with this manuscript. 


\section{References}

1. Basson M (2008) Cardiovascular disease. Nature 451:903

2. Rumble JR, Cooper ME, Soulis T et al (1997) Vascular hypertrophy in experimental diabetes. Role of advanced glycation end products. J Clin Invest 99:1016-1027

3. Cao ZM, Hulthen UL, Allen TJ, Cooper ME (1998) Angiotensin converting enzyme inhibition and calcium antagonism attenuate streptozotocin-diabetes-associated mesenteric vascular hypertrophy independently of their hypotensive action. J Hypertens 16:793-799

4. Candido R, Jandeleit-Dahm KA, Cao Z et al (2002) Prevention of accelerated atherosclerosis by angiotensin-converting enzyme inhibition in diabetic apolipoprotein E-deficient mice. Circulation 106:246-253

5. Candido R, Allen TJ, Lassila M et al (2004) Irbesartan but not amlodipine suppresses diabetes-associated atherosclerosis. Circulation 109:1536-1542

6. Ruiz-Ortega M, Rodriguez-Vita J, Sanchez-Lopez E, Carvajal G, Egido J (2007) TGF-beta signaling in vascular fibrosis. Cardiovasc Res 74:196-206

7. Leask A, Abraham DJ (2004) TGF-beta signaling and the fibrotic response. Faseb J 18:816-827

8. Sorescu D (2006) Smad3 mediates angiotensin II- and TGF-beta1induced vascular fibrosis: Smad3 thickens the plot. Circ Res 98: 988-989

9. Hayden MR, Tyagi SC (2000) Arteriogenesis: angiogenesis within unstable atherosclerotic plaque - interactions with extracellular matrix. Curr Interv Cardiol Rep 2:218-227

10. Schmidt A, Lorkowski S, Seidler D, Breithardt G, Buddecke E (2006) TGF-betal generates a specific multicomponent extracellular matrix in human coronary SMC. Eur J Clin Invest 36:473-482

11. Rudijanto A (2007) The role of vascular smooth muscle cells on the pathogenesis of atherosclerosis. Acta Med Indones 39:86-93

12. Chai Z, Sarcevic B, Mawson A, Toh BH (2001) SET-related cell division autoantigen-1 (CDA1) arrests cell growth. J Biol Chem 276:33665-33674

13. Tu Y, Wu W, Wu $\mathrm{T}$ et al (2007) Antiproliferative autoantigen CDA1 transcriptionally upregulates $\mathrm{p} 21 \mathrm{Wafl} / \mathrm{Cip} 1$ by activating $\mathrm{p} 53$ and MEK/ERK1/2 MAPK pathways. J Biol Chem 282:11722-11731

14. Ito T, Tsukumo S, Suzuki N et al (2004) A constitutively active arylhydrocarbon receptor induces growth inhibition of jurkat $\mathrm{T}$ cells through changes in the expression of genes related to apoptosis and cell cycle arrest. J Biol Chem 279:25204-25210

15. Radke JR, Donald RG, Eibs A et al (2006) Changes in the expression of human cell division autoantigen-1 influence Toxoplasma gondii growth and development. PLoS Pathog 2:e105

16. Datto MB, Li Y, Panus JF, Howe DJ, Xiong Y, Wang XF (1995) Transforming growth factor beta induces the cyclin-dependent kinase inhibitor p21 through a p53-independent mechanism. Proc Natl Acad Sci USA 92:5545-5549

17. Datto MB, Yu Y, Wang X-F (1995) Functional analysis of the transforming growth factor beta responsive elements in the WAF1/ Cip1/p21 promoter. J Biol Chem 270:28623-28628

18. Hu PP, Shen X, Huang D, Liu Y, Counter C, Wang XF (1999) The MEK pathway is required for stimulation of $\mathrm{p} 21$ (WAF1/CIP1) by transforming growth factor-beta. J Biol Chem 274:35381-35387

19. Kim YK, Bae GU, Kang JK et al (2006) Cooperation of $\mathrm{H}_{2} \mathrm{O}_{2^{-}}$ mediated ERK activation with Smad pathway in TGF-beta1 induction of p21WAF1/Cip1. Cell Signal 18:236-243

20. Goldberg HJ, Huszar T, Mozes MM, Rosivall L, Mucsi I (2002) Overexpression of the type II transforming growth factor-beta receptor inhibits fibroblasts proliferation and activates extracellu- lar signal regulated kinase and c-Jun N-terminal kinase. Cell Biol Int 26:165-174

21. Lee MK, Pardoux C, Hall MC et al (2007) TGF-beta activates Erk MAP kinase signalling through direct phosphorylation of ShcA. Embo J 26:3957-3967

22. Ozbun LL, Martinez A, Angdisen J et al (2003) Differentially expressed nucleolar TGF-betal target (DENTT) in mouse development. Dev Dyn 226:491-511

23. Ozbun LL, Martinez A, Jakowlew SB (2005) Differentially expressed nucleolar TGF-betal target (DENTT) shows tissuespecific nuclear and cytoplasmic localization and increases TGFbeta1-responsive transcription in primates. Biochim Biophys Acta 1728:163-180

24. Ozbun LL, You L, Kiang S, Angdisen J, Martinez A, Jakowlew SB (2001) Identification of differentially expressed nucleolar TGF-beta1 target (DENTT) in human lung cancer cells that is a new member of the TSPY/SET/NAP-1 superfamily. Genomics 73:179-193

25. Martinez A, Ozbun LL, Angdisen J, Jakowlew SB (2002) Expression of differentially expressed nucleolar transforming growth factor-betal target (DENTT) in adult mouse tissues. Dev Dyn 224:186-199

26. Yokoyama H, Deckert T (1996) Central role of TGF-beta in the pathogenesis of diabetic nephropathy and macrovascular complications: a hypothesis. Diabet Med 13:313-320

27. Twigg SM, Cooper ME (2004) The time has come to target connective tissue growth factor in diabetic complications. Diabetologia 47:965-968

28. Wrana JL, Attisano L, Carcamo J et al (1992) TGF beta signals through a heteromeric protein kinase receptor complex. Cell 71:1003-1014

29. Dennler S, Itoh S, Vivien D, ten Dijke P, Huet S, Gauthier JM (1998) Direct binding of Smad3 and Smad4 to critical TGF betainducible elements in the promoter of human plasminogen activator inhibitor-type 1 gene. Embo J 17:3091-3100

30. Ling S, Dai A, Dilley RJ et al (2004) Endogenous estrogen deficiency reduces proliferation and enhances apoptosis-related death in vascular smooth muscle cells: insights from the aromatase-knockout mouse. Circulation 109:537-543

31. Park L, Raman KG, Lee KJ et al (1998) Suppression of accelerated diabetic atherosclerosis by the soluble receptor for advanced glycation endproducts. Nat Med 4:1025-1031

32. Hsueh W, Abel ED, Breslow JL et al (2007) Recipes for creating animal models of diabetic cardiovascular disease. Circ Res 100:1415-1427

33. Krege JH, Hodgin JB, Hagaman JR, Smithies O (1995) A noninvasive computerized tail-cuff system for measuring blood pressure in mice. Hypertension 25:1111-1115

34. Soro-Paavonen A, Watson AM, Li J et al (2008) Receptor for advanced glycation end products (RAGE) deficiency attenuates the development of atherosclerosis in diabetes. Diabetes 57:2461-2469

35. Lassila M, Allen TJ, Cao Z et al (2004) Imatinib attenuates diabetes-associated atherosclerosis. Arterioscler Thromb Vasc Biol 24:935-942

36. Calkin AC, Forbes JM, Smith CM et al (2005) Rosiglitazone attenuates atherosclerosis in a model of insulin insufficiency independent of its metabolic effects. Arterioscler Thromb Vasc Biol 25:1903-1909

37. Wang W, Huang XR, Canlas E et al (2006) Essential role of Smad3 in angiotensin II-induced vascular fibrosis. Circ Res 98:1032-1039

38. Wang A, Ziyadeh FN, Lee EY et al (2007) Interference with TGFbeta signaling by Smad3-knockout in mice limits diabetic 
glomerulosclerosis without affecting albuminuria. Am J Physiol Renal Physiol 293:F1657-F1665

39. Li JH, Huang XR, Zhu HJ, Johnson R, Lan HY (2003) Role of TGF-beta signaling in extracellular matrix production under high glucose conditions. Kidney Int 63:2010-2019

40. Macias-Silva M, Abdollah S, Hoodless PA, Pirone R, Attisano L, Wrana JL (1996) MADR2 is a substrate of the TGFbeta receptor and its phosphorylation is required for nuclear accumulation and signaling. Cell 87:1215-1224

41. Liu X, Sun Y, Constantinescu SN, Karam E, Weinberg RA, Lodish HF (1997) Transforming growth factor beta-induced phosphorylation of Smad3 is required for growth inhibition and transcriptional induction in epithelial cells. Proc Natl Acad Sci USA 94:10669-10674

42. Li JH, Wang W, Huang XR et al (2004) Advanced glycation end products induce tubular epithelial-myofibroblast transition through the RAGE-ERK1/2 MAP kinase signaling pathway. Am J Pathol 164:1389-1397

43. Li JH, Huang XR, Zhu HJ et al (2004) Advanced glycation end products activate Smad signaling via TGF-beta-dependent and independent mechanisms: implications for diabetic renal and vascular disease. FASEB J 18:176-178 